\title{
CAPACITAÇÃO DO FAMILIAR CUIDADOR NA ADESÃO À PREVENÇÃO E AO CONTROLE DA HIPERTENSÃO ARTERIAL
}

\section{Training of the family caregiver for adherence to prevention and control of arterial hypertension}

\section{Capacitación del cuidador familiar para la adhesión de la prevención y el control de la hipertensión arterial}

\author{
Amábili Couto Teixeira de Aguiar \\ Hospital Geral Dr. Waldemar Alcântara - HGWA - Fortaleza (CE) - Brasil
}

Zélia Maria de Sousa Araújo Santos

Universidade de Fortaleza - UNIFOR - Fortaleza (CE) - Brasil

Kátia Alves Ferreira Rodrigues

Núcleo de Apoio a Saúde da Família - NASF - Eusébio (CE) - Brasil

Paula Dayanna Sousa dos Santos

Universidade de Fortaleza - UNIFOR - Fortaleza (CE) - Brasil

\section{José Iran das Chagas de Oliveira Júnior}

Secretaria Estadual de Saúde - SESA - Fortaleza (CE) - Brasil

\section{Karla Maria Carneiro Rolim}

Universidade de Fortaleza - UNIFOR - Fortaleza (CE) - Brasil

\section{RESUMO}

Objetivo: Analisar o impacto de uma tecnologia educativa em saúde na capacitação do familiar cuidador no controle da hipertensão arterial e na prevenção do risco deste agravo. Métodos: Pesquisa participante com a aplicação de uma Tecnologia Educativa em Saúde (TES) - Capacitação do familiar cuidador na adesão à prevenção e/ou controle da hipertensão arterial, com um grupo de oito familiares cuidadores (FC), de um Serviço de Assistência Domiciliar de um hospital público de Fortaleza, Ceará, realizada entre julho e outubro de 2016. Resultados: Na primeira entrevista, observou-se que os FC revelaram algum conhecimento sobre hipertensão arterial (HA), modo de descoberta ou diagnóstico, fatores de risco, cronicidade e risco/gravidade. Após a aplicação da TES, ocorreu a apreensão do conhecimento acerca das condutas de controle da HA e de prevenção dos seus fatores de risco, embora de modo irregular sob os aspectos quantitativos e qualitativos em todas as condutas. As experiências vivenciadas pelos FC com a TES emergiram vários significados: incentivo para o autocuidado e para o cuidado do familiar; emponderamento para o cuidado; valorização da saúde; e adoção de hábitos saudáveis. Conclusão: A aplicação da TES com os familiares cuidadores promoveu o envolvimento no autocuidado, no cuidado na família e na comunidade, difundindo o saber construído.

Descritores: Hipertensão; Educação em saúde; Cuidadores.

\section{ABSTRACT}

Objective: To analyze the impact of a health education technology on the training of the family caregiver for the control and prevention of arterial hypertension. Methods: Participant observation was used along with a Health Education Technology (HET) - Training of the family caregiver for adherence to prevention and/or control of arterial hypertension - with a group of eight family caregivers (FC) of a Home Care Service of a public hospital in Fortaleza, Ceará, between July and October 2016. Results: In the first interview, the FC demonstrated some knowledge about arterial hypertension (AH), discovery or diagnosis method, risk factors, chronicity and risk/severity. After the use of HET, they acquired knowledge about the conduct related to AH control and prevention of its risk factors; however, knowledge acquisition occurred in an irregular manner regarding the quantitative and qualitative aspects of the conduct. FC's experiences with the HET gave rise to several meanings: self-care and family care 
encouragement; empowerment for care; health enhancement; and adoption of healthy habits. Conclusion: The use of the HET with family caregivers promoted involvement in self-care, family care and community care, thus disseminating the knowledge built.

Descriptors: Hypertension; Health Education; Caregivers.

\section{RESUMEN}

Objetivo: Analizar el impacto de una tecnología educativa en salud para la capacitación del cuidador familiar para el control de la hipertensión arterial y la prevención del riesgo de ese agravio. Métodos: Investigación participante con la aplicación de una Tecnología Educativa en Salud (TES) - Capacitación del cuidador familiar para la adhesión de la prevención y/o del control de la hipertensión arterial con un grupo de ocho cuidadores familiares (CF) de un Servicio de Atención Domiciliaria de un hospital público de Fortaleza, Ceará, realizada entre julio y octubre de 2016. Resultados: Se observó en la primera entrevista que los CF revelaron algún conocimiento sobre la hipertensión arterial (HA), el modo de descubrimiento del diagnóstico de la enfermedad, los factores de riesgo, la cronicidad y el riesgo/la gravedad. Después de la aplicación de la TES, se dio la aprensión del conocimiento sobre las conductas del control de la HA y de la prevención de sus factores de riesgo, aunque de modo irregular bajo los aspectos cuantitativos y cualitativos de todas las conductas. Emergieron varios significados de las experiencias vividas por los CF con las TES: el incentivo para el autocuidado y para el cuidado del familiar, el empoderamiento para el cuidado, la valorización de la salud y la adopción de hábitos saludables. Conclusión: La aplicación de la TES con los cuidadores familiares promovió el envolvimiento para el autocuidado, el cuidado con la familia y la comunidad difundiendo el saber que ha sido construido.

Descriptores: Hipertensión; Educación en Salud; Cuidadores.

\section{INTRODUÇÃO}

A hipertensão arterial sistêmica (HAS) é uma condição clínica multifatorial caracterizada por níveis elevados e sustentados de pressão arterial (PA) maior ou igual a $140 \mathrm{e} / \mathrm{ou} 90 \mathrm{mmHg}$. Atinge grande parte da população mundial e, quando não tratada adequadamente, traz grandes e graves complicações aos seus portadores. Partes destas alterações são decorrentes da não adesão ao tratamento ou realizado de forma inadequada. Associa-se com frequência a alterações metabólicas, funcionais e/ou estruturais de órgãos alvos ${ }^{(1)}$.

É agravada pela presença de outros fatores de risco (FR), como obesidade abdominal, dislipidemia, intolerância à glicose e diabetes mellitus (DM). Associa-se independentemente com eventos cardiovasculares (CV) como morte súbita, acidente vascular encefálico, insuficiência cardíaca, infarto agudo do miocárdio, doença arterial periférica e doença renal crônica, fatais e não fatais. As complicações ocorrem por comprometimento de órgãos-alvo (rins, coração, encéfalo e vasos sanguíneos) $)^{(1)}$.

Trata-se da principal causa de óbito prevenível no mundo, sendo responsável por 13,0\% das mortes. Em 2000, a prevalência mundial era estimada em torno de $26,0 \%$, devendo passar para $29,0 \%$ em 2025 , considerando apenas o aumento populacional e a composição etária. Esse percentual equivale a aproximadamente 1,56 bilhão de pessoas afetadas. Na América Latina, a HAS afeta mais de um terço da população(2).

A atenção à pessoa com HAS considerando-se a interação e a participação familiar, tem sido abordada e destacada por inúmeros estudos, onde, cada vez mais, encontram-se trabalhos que evidenciam o sucesso que pode resultar da associação entre o cuidado familiar e o cuidado profissional(3).

A família é primordial no cuidado dos seus membros, tanto em condições de saúde como doença, e sua importância tem sido relacionada a uma maior adesão ao tratamento por indivíduos com algum problema de saúde, especialmente quando a mesma é incluída como participante no processo saúde-doença, já que ela contribui de forma substancial na promoção da saúde ${ }^{(4)}$.

O contexto familiar é o primeiro espaço de identificação e explicação do adoecimento de seus membros e onde os fenômenos da saúde e da doença adquirem maior relevância. O impacto da doença recai sobre todos os membros da família, assim como a interação familiar exerce influência sobre a sua cura ${ }^{(5)}$.

Os membros da família podem apresentar-se tímidos e inseguros para se envolverem no cuidado de um familiar doente, mas se tiverem apoio do Enfermeiro, percebe-se uma aceitação destes no cuidado direto. O Enfermeiro deve estar atento às dificuldades inerentes ao processo de adaptação da família na participação do cuidado ao membro doente, intervindo sempre que se fizer necessário(6).

A família vem ficando à margem no planejamento dos cuidados pelas Equipes de Saúde (EqS) ou Equipes Saúde da Família (EqSF), cujo foco tem sido o indivíduo, na condição de usuário que se encontra hospitalizado 
ou dependente no domicílio, em áreas adstritas a estas equipes. Entende-se, preliminarmente, que, ao não incluir a família em seu plano de cuidados, não há avanço na proposta de um modelo assistencial orientado segundo os preceitos da promoção da saúde e pelo princípio da integralidade ${ }^{(7)}$.

Então, a EqS do SAD deve prestar cuidados também aos FC, principalmente investindo na prevenção e promoção da saúde. Esses cuidados fundamentar-se-ão na educação em saúde (ES), porque os empoderará para o autocuidado com sua saúde e seu bem-estar. A ES possibilita a emancipação do sujeito, fortalecendo o vínculo entre democracia e educação. É necessário conhecer o outro e adentrar as práticas cotidianas para desenvolver habilidades que possibilitem trocas efetivas a fim de buscar uma inserção da pessoa na coletividade. Além disso, deve ser vista como direito social, constituindo-se historicamente um pré-requisito para a expansão dos demais direitos. A educação de sujeitos, voltada para a saúde, na perspectiva crítica, requer a compreensão de que a educação ocorre na forma de processo, em que aprender implica construir e não adquirir conhecimentos; significa desenvolver habilidades pessoais e sociais, e não adaptar ou reproduzir comportamentos ${ }^{(8)}$.

O uso de tecnologias da informação e comunicação (TIC) desenvolve-se rapidamente e são empregadas com o objetivo de aumentar a adesão ao tratamento de patologias crônicas como a HAS. De grande importância é a utilização das tecnologias com a finalidade de favorecer a comunicação entre profissionais de saúde e os usuários. São centradas nos cuidados à pessoa e por meio de informações clínicas sobre a patologia. Objetiva tornar a pessoa mais consciente acerca do caráter assintomático e da natureza crônica da HAS e auxilia também no estímulo motivacional para a adesão ao tratamento farmacológico e não farmacológico. Educação efetiva deve ser realizada de forma contínua com a finalidade de resultar em mudanças comportamentais. Há um grande desafio em integrar todos os profissionais de saúde com os usuários para que ocorram estas mudanças ${ }^{(9)}$. Mediante da problemática da HAS para a Saúde Pública, e em particular o estilo de vida dos FC susceptível ao risco da HAS e de outros agravos, optou-se por este estudo, com o objetivo de analisar o impacto de uma tecnologia educativa em saúde na capacitação do familiar cuidador no controle da hipertensão arterial sistêmica e na prevenção do risco deste agravo.

\section{MÉTODOS}

Trata-se de uma pesquisa participante realizada no Serviço de Assistência Domiciliar (SAD) de um hospital público de Fortaleza, Ceará, Brasil, cuja missão é prestar serviços de saúde com qualidade, em nível secundário, aos usuários do SUS referenciados pelas Centrais de Leitos do Estado do Ceará e deste município, desenvolvida no período de julho a outubro de 2016.

Participaram do estudo 08 (oito) familiares cuidadores $(F C$ ), sendo quatro com hipertensão arterial sistêmica (HAS) e quatro sem HAS, integrantes do SAD pertencente à instituição supracitada que assistiam as pessoas com doenças crônicas dependentes de cuidados, independentemente da idade, escolaridade, cor, etc. Denominou-se FC aquele membro da família que mais se destaca no cuidado com a pessoa acometida pela HAS. Inicialmente selecionou-se um grupo com 20 (vinte) participantes, considerando a possibilidade de evasão ou desistência. No entanto, 12 (doze) desistiram pelos seguintes motivos: gravidade do familiar assistido, e falta de substituição no cuidado do familiar.

Ao longo dos anos de experiência na prestação de cuidados a pessoas com doenças crônicas, inscritas no Serviço de Assistência Domiciliar (SAD) em Fortaleza, Ceará, tem-se observado familiares cuidadores (FC) hipertensos não aderentes às condutas de controle da HAS, e aqueles normotensos com estilo de vida compatível com o surgimento deste agravo, e quando indagadas sobe este fato, frequentemente, há referência à priorização do tempo dedicado ao cuidado do familiar doente. Contudo, se percebe também déficit de conhecimento relacionado à HAS, às condutas de controle e de prevenção dos fatores e risco.

A Tecnologia Educativa em Saúde (TES) intitulada "Capacitação do familiar cuidador na adesão à prevenção e/ ou controle da hipertensão arterial" foi elaborada com base nos pressupostos da educação em saúde. A aplicação da TES consistiu de 10 (dez) encontros semanais, com duração média de sessenta minutos. Ressalta-se que a determinação dos dias da semana e horário para os encontros foram de acordo com os FC, de modo a não prejudicar a sua rotina diária.

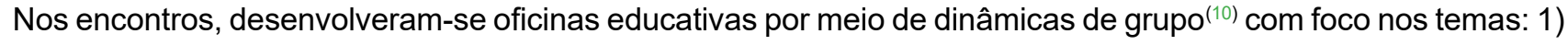
resgate com socialização do saber construído pela experiência relacionado com a $\mathrm{HAe}$ as medidas de prevenção $\mathrm{e}$ de controle da HA; 2) discussão sobre a HA como problema de Saúde Pública, e sobre cronicidade, assintomatologia, fatores de risco, diagnóstico e risco/gravidade; e 3) condutas de controle da HA e as preventivas do risco da HAS: alimentação saudável, prática regular do exercício físico, prevenção e combate do alcoolismo, tabagismo e uso de 
drogas ilícitas, gerenciamento do estresse, uso regular da medicação antihipertensiva pelos FC com HA, bem como a guarda, conservação, efeitos terapêuticos e adversos, e desconfortos do esquema medicamentoso. Encerraram-se os encontros com a realização do feedback sobre a participação dos FC e uma autoavaliação destes, seguidas da elaboração de um "Plano de Ação" com vista à adesão às condutas de prevenção e/ou de controle da HA.

Coletaram-se os dados por meio das técnicas: entrevista e observação participante. Gravaram-se as entrevistas conforme o consentimento prévio dos FC, e complementou-se com as informações registradas durante os encontros em um diário de campo. Os FC participaram de uma reunião aprazada em consenso com a direção da instituição e da coordenação do SAD, na qual foram expostos a natureza e os objetivos da pesquisa, e confirmou-se a anuência quanto à participação no estudo, por meio da assinatura no Termo de Consentimento Livre e Esclarecido (TCLE), e o compromisso com a participação no estudo. Realizaram-se duas entrevistas: a primeira, anterior à aplicação da TES, cujo roteiro conteve os dados sociodemográficos, conhecimento sobre a HAS, condutas preventivas e de controle; e a segunda, com a questão norteadora: Comente sobre a o impacto desses encontros na sua capacitação para a adesão às condutas preventivas e/ou de controle da HA?

As informações foram coletadas a partir da técnica de análise de conteúdo(11), conforme as etapas para a análise e interpretação dos dados coletados: Pré-análise - leitura exaustiva das entrevistas e das anotações no diário de campo; Exploração do material - após a leitura, relacionaram-se as categorias analíticas, e posteriormente selecionou-se os trechos de relatos, considerados relevantes; e Tratamento dos resultados - com os resultados obtidos, procedeu-se à interpretação, nos fundamentando nos pressupostos da educação em saúde, e na literatura selecionada.

Desenvolveu-se este estudo com base na Resolução 466/12 da Comissão Nacional de Ética em Pesquisa $(\mathrm{CONEP} / \mathrm{CNS} / \mathrm{MS})^{(12)}$, que regulamenta a pesquisa com seres humanos, aprovado sob Parecer $\mathrm{n}^{\circ} .1 .432 .244$ pelo comitê de ética da Universidade de Fortaleza. Identificaram-se os participantes pelas letras FCH (FC com HAS) e FCN (FC sem HAS), seguidas da numeração relativa ao número de participantes (FCH1 a FCH4, e FCN1 a FCN4).

\section{RESULTADOS E DISCUSSÃO}

Para o processamento da análise, agruparam-se os resultados nas categorias: caracterização sociodemográfica dos familiares cuidadores; e análise do impacto da Tecnologia Educativa em Saúde "Capacitação do familiar cuidador na adesão à prevenção e/ou controle da hipertensão arterial".

\section{Caracterização sociodemográfica dos familiares cuidadores}

Os familiares cuidadores (FC) eram do sexo feminino; sete adultas (32 e 47 anos de idade) e uma idosa (60 anos); quanto ao estado civil - quatro casadas, duas separadas, uma solteira e uma viúva; em relação á prática religiosa - quatro católicas e quatro evangélicas; sete nasceram em outros municípios do Estado do Ceará; três cursaram o ensino médio, três o ensino fundamental e duas não concluíram o ensino fundamental; três habitavam em imóvel próprio e cinco em imóvel alugado; a renda mensal individual era em torno de um salário mínimo (SM) vigente $(\mathrm{R} \$ 880,00)$, e a familiar era de até quatro SM.

O vínculo família do FC, incluía: uma filha, uma neta, cinco mães, e uma sogra; seis famílias eram do tipo nuclear e duas do tipo ramificada. Dentre aquelas que tinham ocupação remunerada fora do domicílio, havia duas costureiras, uma manicure, uma empregada doméstica e uma artesã.

Os FC do estudo eram mulheres. Este achado corrobora a literatura(5.) ao fazer uma reflexão sobre as práticas do cuidar, historicizando o que considera próprio da natureza feminina, que é a tarefa do cuidar e concorda com outros estudos $^{(13-15)}$ sobre familiares cuidadores, evidenciando que o grupo é constituído predominantemente por mulheres.

A baixa escolaridade apresentada pelas mulheres da presente pesquisa consiste tanto em um fator de risco para HAS quanto em empecilho para adequada adesão ao tratamento e controle. Estudo epidemiológico ${ }^{(16)}$ evidenciou variações importantes da HAS quanto à escolaridade, sendo a prevalência de $44 \%$ entre os que não completaram o ensino médio e de $28,4 \%$ entre os participantes que tinham pós-graduação. Já em um estudo que avaliou a relação entre adesão ao tratamento ${ }^{(17)}$, o baixo nível educacional tem sido apontado como um dos mais importantes entraves.

\section{Repercussão da aplicação da Tecnologia Educativa em Saúde "Capacitação do familiar cuidador na adesão à prevenção e/ou controle da hipertensão arterial"}

Admite-se como impacto da TES mediante os relatos do FC: esclarecimento de dúvidas sobre o tratamento da hipertensão arterial (HA); adaptação e mudanças nos hábitos; envolvimento no autocuidado; conscientização do 
risco da $\mathrm{HA}$ e incentivo aos familiares sobre a prevenção; e agente multiplicadora do saber adquirido na família e na comunidade.

\section{Esclarecimentos de dúvidas sobre condutas de controle da HA:}

"[...] tirei minhas dúvidas em relação a alimentação, exercício físico, aprendi que é importante tomar a medicação direitinho e fiquei mais atenta sobre isso [...]." (FCH1, 47 anos)

Mediante o relato, percebeu-se o acréscimo de informações sobre as condutas de controle, e o despertar para o compromisso com o controle da HA.

\section{Adaptação e mudanças nos hábitos:}

"[...] mudou muitas coisas, eu estou tentando me adaptar, me alimentando melhor [...] o uso do sal que eu era acostumada, e achava que era normal e bom, aprendi que não é, cortei o refrigerante e coisas gordurosas, agora faço no Grill [...]." (FCN2, 32 anos)

As ações educativas favoreceram ao surgimento de mudança nos hábitos para um estilo de vida saudável com vista ao controle dos fatores de risco da HA.

A Promoção da Saúde é uma estratégia de articulação transversal, cujo objetivo é promover a qualidade de vida e reduzir os riscos à saúde relacionados aos seus determinantes e condicionantes, por meio de ações que possibilitem responder às necessidades sociais da população(18). Embasado neste conceito, o Ministério da Saúde (MS), propõe a reorientação dos serviços de saúde, ampliando seu escopo para incluir intervenções sobre as condições de vida e apoiando escolhas saudáveis por parte dos sujeitos e das coletividades ${ }^{(19)}$.

\section{Envolvimento no autocuidado:}

"[...] me ajudou a ter mais cuidado com a comida, estou cuidando para não comer sal, a fazer meus exercícios físicos, a cuidar mais de mim [...] porque apesar de eu cuidar do meu genro eu também tenho que cuidar de $\operatorname{mim}[\ldots] . "$ (FCH5, 60 anos)

A TES possibilitou ao FC o envolvimento no seu autocuidado, comprometendo-se com a promoção de sua saúde.

Nem o ensino depende exclusivamente do educador, nem o aprendizado é exclusivo do educando. O fazer educativo dialógico requer que o educador admita não possuir todo o saber e que o educando compreenda a importância da sua contribuição a construção do conhecimento(20).

\section{Conscientização do risco da HA e incentivo aos familiares sobre a prevenção:}

"[...] eu agora estou fazendo as comidas com mais consciência, diminui o sal e a gordura, estou fazendo caminhada e estou incentivando minhas cunhadas e minha irmã [...]." (FCN7, 37 anos)

Percebe-se a mudança de comportamento e a socialização das experiências vivenciadas nas oficinas entre os familiares.

Consideram-se, como aspectos fundamentais, o desenvolvimento de habilidades que permitam ao portador de uma doença crônica uma postura mais crítica diante dos seus problemas de saúde, com capacidade de exercer um controle sobre si mesmo e o ambiente em que vivem ${ }^{(21)}$.

Tomar consciência dos riscos é mais do que saber o que se passa ao seu redor, o homem reage aos desafios que lhe apresenta seu contexto de vida, ele se cria, se realiza como sujeito, reflete, critica, inventa(2).

Agente multiplicador do saber adquirido na família e na comunidade:

"[...] com certeza me ajudou muito, mudei, fiquei mais segura em saber como fazer melhor os alimentos e posso até ensinar minha família e meus vizinhos [...]." (FCH8, 38 anos)

Visualizou-se a essência das ações educativas na mudança comportamental, em que houve o empoderamento, tornando-se agente multiplicador do saber construído junto aos familiares e à comunidade.

A capacitação de cuidadores serve como um instrumento de apoio aos profissionais envolvidos na assistência a si, aos pacientes e a suas famílias, permitindo que atuem como multiplicadores dos conhecimentos adquiridos ${ }^{(23)}$. 


\section{CONSIDERAÇÕES FINAIS}

Os Familiares cuidadores (FC) eram do sexo feminino e revelaram algum conhecimento sobre a hipertensão arterial sistêmica (HAS) e seu tratamento, mas superficial e fragmentado e adquirido pela experiência com o próprio agravo, de outrem, ou ainda de orientações recebidas pela Equipe de Saúde (EqS) do Serviço de Atenção Domiciliar (SAD). Entretanto, a aplicação da Tecnologia Educativa em Saúde (TES) repercutiu favoravelmente no seu empoderamento, comprometendo-se com o autocuidado, tornando-se, de certo modo, agente de autocuidado e multiplicador das ações de autocuidado na família e na comunidade.

Contudo, é necessário capacitar os FC para cuidar do outro, e de si mesmo, ou seja, cuidar de quem cuida. Torna-se necessário apoiá-los e incentivá-los a encontrar meios que possam reduzir os fatores de risco e controlar a HAS. Ao construírem o conhecimento em relação à doença, os FC puderam se empoderar e realizar mudanças no cotidiano estendendo o cuidado com a própria saúde para o restante da família e até vizinhos e amigos. Portanto, diante do que foi discutido e considerado, conclui-se que a forma mais verdadeira de parceria entre pesquisador $\mathrm{e}$ FC é o da Educação em Saúde. Por meio desta parceria, os FC não serão meros espectadores, mas protagonistas da própria saúde, agindo de maneira tal que se torne multiplicador dessas ações preventivas e de controle, sensibilizando a comunidade que os rodeia.

\section{REFERÊNCIAS}

1. Sociedade Brasileira de Cardiologia. VII Diretriz Brasileira de Hipertensão Arterial. Arq Bras Cardiol. 2016;107(13 Supl 2):1-103.

2. Karakurt $\mathrm{P}$, Kasilçi M. Factors affecting medication adherence in patients with hypertension. J Vasc Nurs. 2012;30(4):118-26.

3. Lopes MCL, Marcon SS. A hipertensão arterial e a família: a necessidade do cuidado familiar. Rev Esc Enferm USP. 2009;43(2):343-50.

4. Squarcini CFR, Silva LWS, Reis JF, Pires EPOR, Tonosaki LMD, Ferreira GA. A pessoa idosa, sua família e a hipertensão arterial: cuidados num programa de treinamento físico aeróbico. Rev Kairós. 2011;14(3):105-25.

5. Martins MM, Fernandes CS, Gonçalves LHT. A família como foco dos cuidados de enfermagem em meio hospitalar: um programa educativo. Rev Bras Enferm. 2012;65(4):685-90.

6. Wright LM, Leahey M. Enfermeiras e famílias. 3. ed. São Paulo: ROCA; 2012.

7. Costa SRD, Castro EAB. Autocuidado do cuidador familiar de adultos ou idosos dependentes após a alta hospitalar. Rev Bras Enferm. 2014;67(6):979-86.

8. Lopes R, Tocantis, FR. Promoção da saúde e a educação crítica. Interface Comun Saúde Educ. 2012;16(40):23546.

9. Santos FPA, Nery AA, Matumoto S. A produção do cuidado a usuários com hipertensão arterial e as tecnologias em saúde. Rev Esc Enferm USP. 2013;47(1):107-14.

10. Jalowitzki M. Vivências para dinâmicas de grupos - a metamorfose do ser a 360. São Paulo: Madras; 2012.

11. Bardin L. Análise de conteúdo. Lisboa: Edições 70; 2014.

12. Brasil. Ministério da Saúde. Conselho Nacional de Saúde. Comissão Nacional de Ética em Pesquisa. Resolução n 466 de 12 de dezembro de 2012, que dispõe sobre a regulamentação de pesquisa envolvendo seres humanos. Brasília: CONEP; 2012.

13. Diniz MAA, Monteiro DQ, Gratão ACM. Educação em saúde para cuidadores informais de idosos. Saúde Transform Soc. 2016:7(1):28-40.

14. Yamashita $\mathrm{CH}$, Amendola F, Alvarenga MRM, Oliveira MAC. Perfil sociodemográfico de cuidadores familiares de pacientes dependentes atendidos por uma unidade de saúde da família no município de São Paulo. Mundo Saúde. 2010,34(1):20-4.

15. Aragão EMS, Nunes RTD. Caracterização do cuidador familiar de afásicos de uma instituição na cidade de Salvador (BA). Rev Baiana Saúde Pública. 2009;33(4):553-60. 
16. Chor D, Ribeiro ALP, Carvalho MS, Duncan BB, Lotufo PA, Nobre AA, et al. Prevalence, awareness, treatment and influence of socioeconomic variables on control of high blood pressure: results of the ELSA-Brasil Study. PLOS One. 2015;10(6):127-382.

17. Noack FK, Cornejo-Contreras G, Castillo LJA. Adherencia al tratamiento farmacológico en pacientes hipertensos del Hospital Comunitario de Yungay, 2012. Rev ANACEM. 2013;7(2):75-8.

18. Rodrigues AAAO, Juliano IA, Melo MLC, Beck CLC, Prestes FC. Processo de interação ensino, serviço e comunidade: a experiência de um PET-Saúde. Rev Bras Educ Méd. 2012;36(1 Supl 2):184-92.

19. Ministério da Saúde (BR), Secretaria de Vigilância em Saúde, Secretaria de Atenção à Saúde. Política Nacional de Promoção da Saúde. $3^{\mathrm{a}}$ ed. Brasília: Ministério da Saúde; 2010.

20. Freire P. Pedagogia da autonomia. São Paulo: Paz e Terra; 2011.

21. Baldissera VDA, Pludo D, Moreira, NM, Garbelini LF, Carvalho MDB. Mudanças vivenciadas por hipertensos após o diagnóstico da doença. Rev Inst Ciênc Saúde. 2008;26(3):304-9.

22. Freire P. Conscientização. São Paulo: Cortez; 1980.

23. Silva RCA, Monteiro GL, Santos AG. O enfermeiro na educação de cuidadores de pacientes com sequelas de acidente vascular cerebral. Rev Atenção Saúde. 2015;13(45):114-20.

\section{Endereço para correspondência:}

Amábili Couto Teixeira de Aguiar

Universidade de Fortaleza - UNIFOR

Programa de Pós-Graduação em Saúde Coletiva - PPGSC

Av. Washington Soares, 1321, Bloco S, Sala 01

Bairro: Edson Queiroz

CEP: 60.811-905 - Fortaleza - CE - Brasil

E-mail: amabilicouto@yahoo.com.br 